\title{
Image Reconstruction method for Human Shape Detection Using One-Dimensional Brightness Distribution Sensor
}

\author{
Shota Nakashima ${ }^{\mathrm{a},}$, Kazuo Haruyama ${ }^{\mathrm{b}}$, Yuhki Kitazono ${ }^{\mathrm{c}}$ \\ ${ }^{a}$ Yamaguchi University, 2-16-1 Tokiwadai, Ube, Yamaguchi, Japan \\ bUbe National College of Technology, 2-14-1 Tokiwadai, Ube, Yamaguchi, Japan \\ ${ }^{\mathrm{c}}$ Kitakyushu National College of Technology, 5-20-1 Shii, Kokuraminami, Kitakyushu, Fukuoka, Japan \\ *Corresponding Author: s-naka@yamaguchi-u.ac.jp
}

\begin{abstract}
In this study, a detection of human shape with privacy-preserving by image reconstruction has been developed. In theory, the sensor can be constructed with a line sensor and cylindrical lens because only a one-dimensional brightness distribution is needed. The proposed sensor obtains a one-dimensional horizontal brightness distribution that is approximately equal to the integration value of each vertical pixel line of the two-dimensional image. We can recognize the human shape by change of the one-dimensional distribution. However, it's difficult to grasp it without the technical knowledge of the proposed system for average person from the distribution. For that reason, the image of human shape is reconstructed by two distributions, although it is impossible to restore the two-dimensional detail texture image correctly. Thus, the privacy is protected. Moreover, the appearance of the proposed sensor is very different from the conventional video camera, so the psychological resistance of having a picture taken is reduced. In this work, we reconstructed the image using one-dimensional brightness distribution obtained by the privacy-preserving sensor, and verified whether a person's shape was able to be detected. The simulation results show that the proposed sensor can detect a present person's state responsively without violating privacy.
\end{abstract}

Keywords: human detection, obrid-sensor, line sensor

\section{Introduction}

Recently, elderly people living alone increased with the trend toward to the nuclear family. They cannot be rescue in case of serious accidents in a restroom and bathroom. To solve this problem, surveillance cameras are convenient but cannot be installed in restrooms and bathrooms because of privacy. Many people feel uncomfortable about video images being recording by cameras even in public places, and in recent years, the preservation of privacy has been requested. Therefore, such a video image is deteriorated by a lot of processing methods for the preservation of privacy $^{(1-3)}$. However, the image may be restored as far as it where it was obtained with a camera even if the image is blurred or encrypted. Moreover, it is not easy to notice the difference between privacy-preserving cameras and normal cameras from external sources. That is why a system for the detection of a person's state and position without taking pictures is needed. For instance, pyroelectric type temperature sensors are generally used to detect a person's presence by infrared rays, but it cannot distinguish the person's state and/or position indoors ${ }^{(4)}$. In order to the problem, we proposed a privacy-preserving sensor for a person localization in a previous work ${ }^{(5)}$, and by using the integration value of each vertical pixel line of the two-dimensional (2-D) image instead of the one-dimensional (1-D) brightness distribution obtained by combining the line sensor and the cylindrical lens, because these two are thought to be approximately same in theory. Simulation results showed that it can confirm a person's presence, position, and movement state, and it also can judge whether person is standing upright or fallen based on a 1-D brightness distribution that does not violate privacy. Images which violate privacy are not restorable in theory because the sensor for the person localization obtains only a 1-D brightness distribution. Then we really developed the new sensor which could detect a 1-D brightness distribution 
without a 2-D image ${ }^{(6)}$. However, person's state cannot be recognized very easy from the 1-D brightness distribution directly. Therefore, we propose a new method which can reconstruct the image from the 1-D brightness distribution obtained by the sensor.

\section{One-Dimensional Brightness Distribution Sensor}

The proposal of a sensor that detect a person's state without invasion of privacy was introduced in our previous work $^{(5)}$. In Ref. (5), we have proposed a privacy-preserving sensor that discriminates between standing and tumbled states of a person by a 1-D brightness distribution of a 2-D image. In theory, the sensor can obtain a 1-D brightness distribution by combining the line sensor and the rod lens (see the section called "Acquisition model of brightness distribution” for details). The 1-D brightness distribution contained the human state and we used the $\operatorname{LVQ}^{(7)}$ as the algorithm for estimating the human state. Furthermore, in order to obtain the optimum parameters of the LVQ, we used the S-System ${ }^{(8-9)}$, which is one of the extended models of $\mathrm{GA}^{(10)}$ and $\mathrm{GP}^{(11)}$. Thus, a privacy-preserving sensor that differentiates between a person who is upright and one who has fallen was made possible without using cameras and vast equipment. In addition, use of the S-System provided good parameters despite a lack of experience and familiarity, and a discrimination circuit was created using LVQ. However, since the device configuration was incomplete at that time, we simulated the brightness distribution from a camera captured image to verify our thinking. Naturally, it is necessary to confirm the effectiveness by using the true proposed sensor. Thus, we actually have developed a one-dimensional brightness distribution sensor (hereinafter referred to as "Obrid-Sensor") for human detection in indoors ${ }^{(6)}$.

\subsection{Basic structure}

The model of the Obrid-sensor is shown in Fig. 1. The sensor constructed was by the rod lens (SAKAI-G rod lens $\varphi 3 \times 110 \mathrm{~mm}$ ) and the CMOS image sensor (Logicool Qcam Pro 9000). The appearance of the Obrid-sensor is shown in Fig. 2. Compare to expensive line sensors, CMOS area sensors are low cost and increasing in sensitivity according to recent rapid advancement in the technology. Therefore, a CMOS image sensor is covered by black covers that have a line shaped window over the center row, so that it behaves

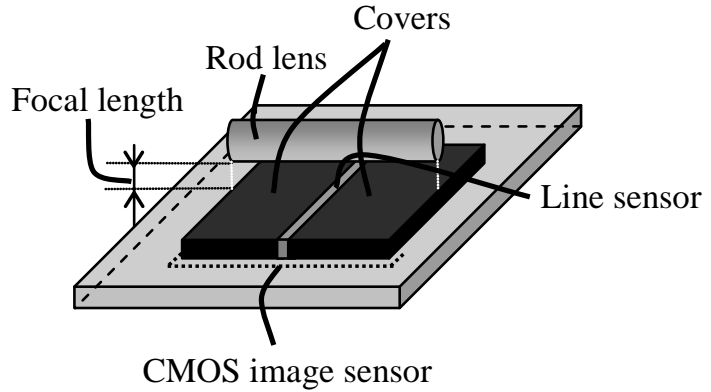

Fig. 1. Construction of the Obrid-Sensor

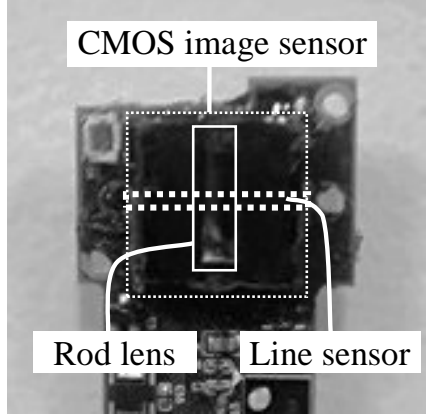

Fig. 2. Appearance of the Obrid-Sensor

as a line sensor. The rod lens has the same properties as the cylindrical lens which is written by Ref. (5).

\subsection{Acquisition model of brightness distribution}

The Obrid-sensor detects a person's state while preserving their privacy. The structure is shown in Fig. 3. Figure 3(a), (b), and (c) show its overall view, side view and top view respectively. As can be seen in Fig. 3(b), when viewed from the side, the rod lens is the shape of a flat panel, and the light radiation from a physical object is input into the sensor even if a vertical position is different. In other words, the distribution of vertical light is integrated. In addition, as can be seen in Fig. 3(c), the rod lens is a convex lens when it is viewed from the top. Therefore, when the physical object's horizontal position is different, the position projected on the sensor will also be different. Summarizing the abovementioned data, as shown in Fig. $3(\mathrm{a})$, the light radiated from the physical object's $\mathrm{R}^{+}, \mathrm{R}, \mathrm{R}^{-}$ enters the $S_{R}$ point of the line sensor that radiated from $\mathrm{C}^{+}$, $\mathrm{C}, \mathrm{C}^{-}$, enters the $\mathrm{S}_{\mathrm{C}}$ point, and $\mathrm{L}^{+}, \mathrm{L}, \mathrm{L}^{-}$enters the $\mathrm{S}_{\mathrm{L}}$ point. In other words, the output from the line sensor indicates how the vertical integration of the light from the object distributes in the horizontal direction. Thus, a privacy-preserving sensor can be achieved without using cameras or using a large scale device. 


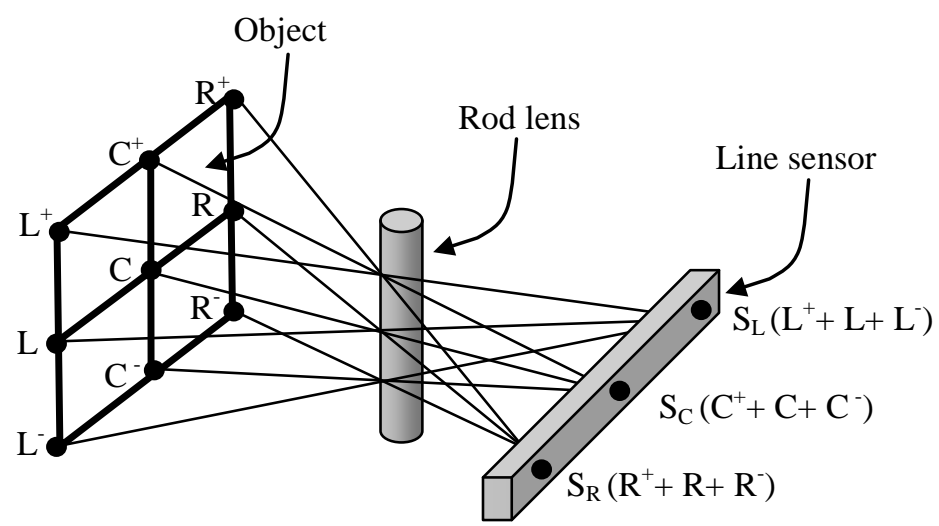

(a) Overall view

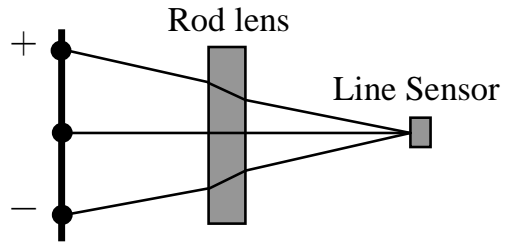

(b) Side view

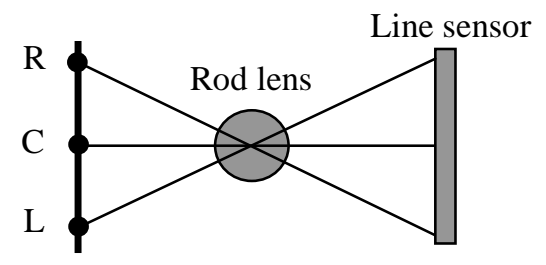

(c) Top view

Fig. 3. Structure of the Obrid-Sensor

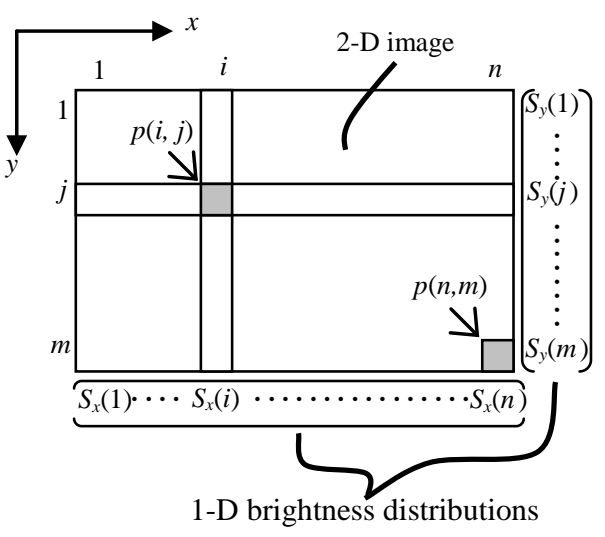

Fig. 4. Relationship between 1-D brightness distributions and 2-D image

\subsection{Relationship between 1-D brightness and 2-D image}

The relationship between the 1-D brightness distributions and the 2-D image is shown in Fig. 4 and Eq. (1).

$$
\left.\begin{array}{l}
S_{x}(i)=\sum_{j=1}^{m} p(i, j) \\
S_{y}(j)=\sum_{i=1}^{n} p(i, j)
\end{array}\right\} \cdots \ldots \ldots \ldots \ldots \ldots \ldots \ldots \ldots(1)
$$

where, $n$ represents the pixel count of the horizontal direction and $m$ represents the pixel count of the vertical direction. Its example is shown in Fig. 5. The horizontal

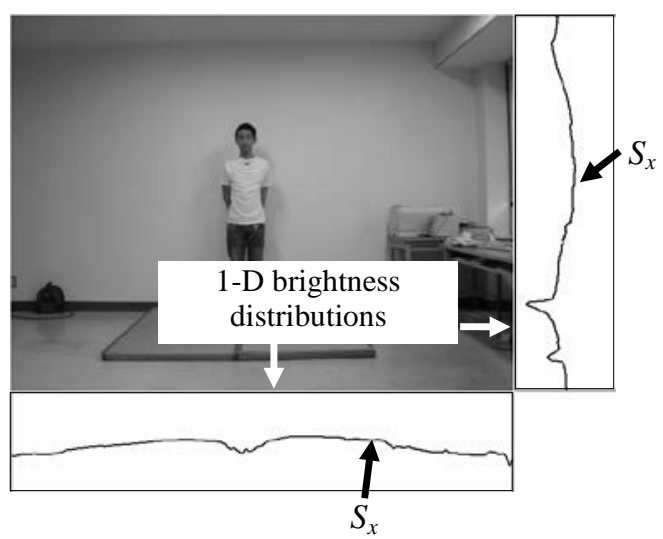

Fig. 5. Example of the value $S_{x}$ and $S_{y}$

and vertical direction distribution in the figure represents the $S_{x}(i)$ and $S_{y}(j)$ obtained by Eq. (1). In this study, $S_{x}(i)$ and $S_{y}(j)$ shown in Eq. (1) are obtained. By rotating the angle of each device by 90 degrees using the two Obrid-sensor are shown in Fig. 1, it is possible to obtain $S_{x}(i)$ and $S_{y}(j)$.

\section{Image Reconstruction}

In order to detect person's situation intuitively, in this research we propose to employ and reorganize the 1-D brightness distribution to 2-D images to estimate the posture of person. The brightness distribution fetched by sensors in vertical and horizontal directions is shown in Fig. 


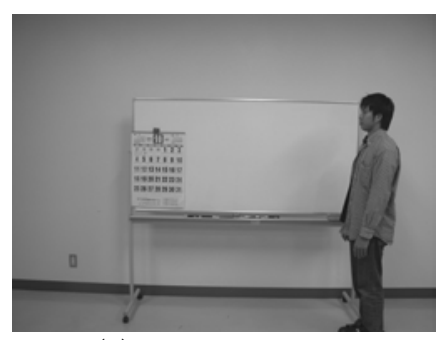

(a) Camera image

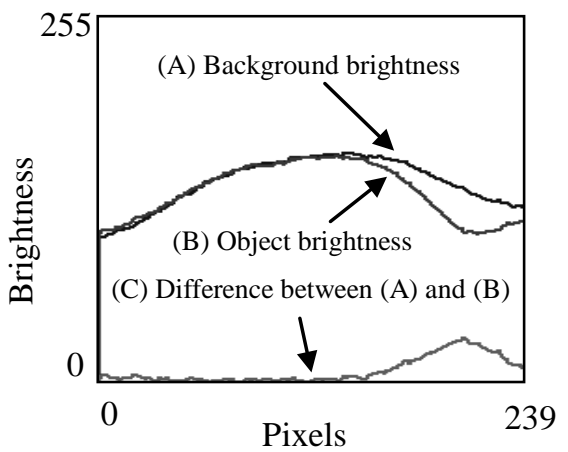

(b) Brightness distributions of (a)

Fig. 6. The 1-D distributions and camera image

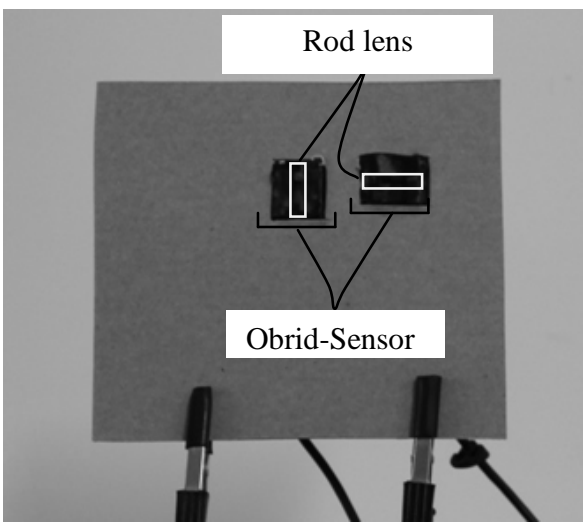

Fig.7. Developed sensor unit

4. In $x$ and $y$ directions of 2-D image, the summary of pixels $p(x, y)$ can be expressed by $S_{x}(i)$ and $S_{y}(j)$ as we see in Eq. (1). As Fig. 5 shows, after obtaining the brightness distribution of background only, the differential of brightness distribution with person can be estimated as 1-D brightness distribution $S_{x}(i)$ and $S_{y}(j)$. The 2-D image can be reconstructed based on the differential of 1-D brightness distribution. In this paper, the novel accumulation reconstructing method is proposed.

When the 1-D brightness distribution $S_{x}(i)$ and $S_{y}(j)$ in $x$ and $y$ directions can be obtained, the pixels $\tilde{p}(i, j)$, which satisfy Eq. (1), can be estimated. The inverse problem is that $m+n$ linear simultaneous equations with $m \times n$ variables cannot get certain solutions.

In the future, it is difficult to use PC in compact sensing systems combined with the algorithms which

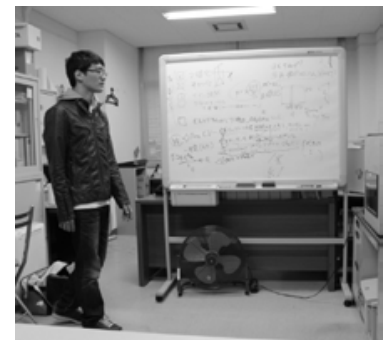

(a) Person in the left side

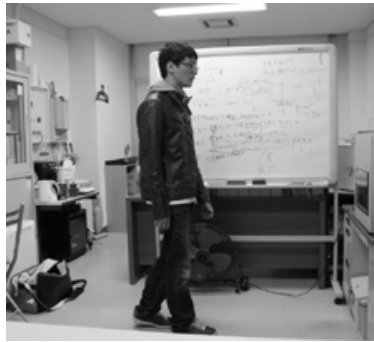

(b) Person in the middle side

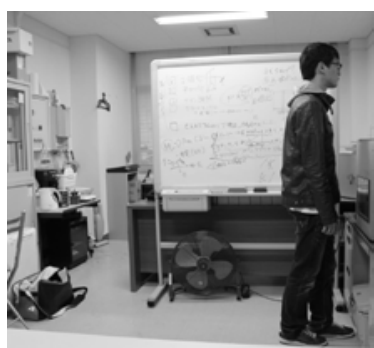

(c) Person in the right side

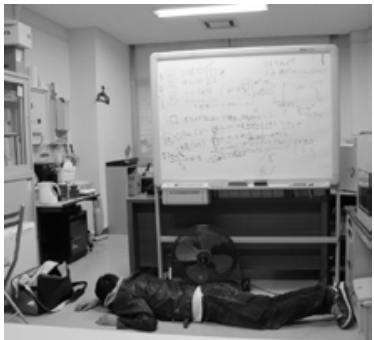

(d) Person in the bottom side

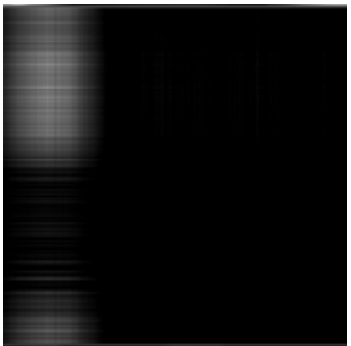

(e) Reconstruction image of (a)

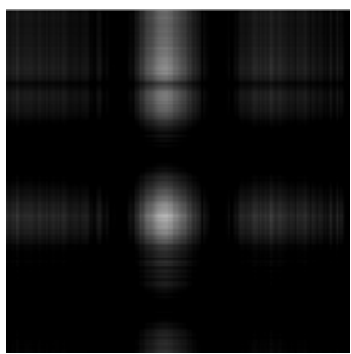

(f) Reconstruction image of (b)

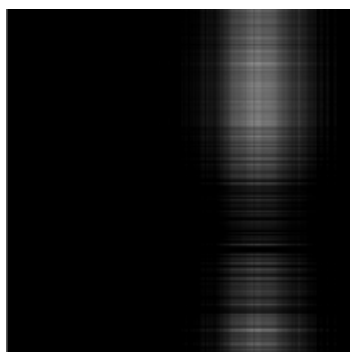

(g) Reconstruction image of (c)

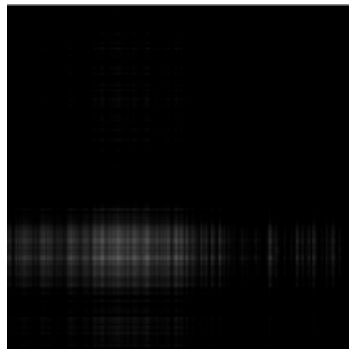

(h) Reconstruction image of (d)
Fig. 8. Camera image and reconstruction image

estimate person's position. Hence, instead of complex algorithms, such as iteration algorithms, the ideal algorithms are expected to be as simple as possible. Therefore, in this paper, the pixels $\tilde{p}(i, j)$ reconstructed according to the 1-D brightness distribution $S_{x}(i)$ and $S_{y}(j)$ will be estimated by Eq. (2).

$$
\tilde{p}(i, j)=\frac{S_{x}(i) \cdot S_{y}(j)}{s}
$$

In Eq. (2), $s$ is the summary of all pixel brightness, expressed by $\sum_{i=1}^{n} S_{x}(i)$ and $\sum_{j=1}^{m} S_{y}(j)$. Since $\sum_{i=1}^{n} S_{x}(i)$ and 
$\sum_{j=1}^{m} S_{y}(j)$ in Eq. (2) equal to $S_{x}(i)$ and $S_{y}(j)$, respectively, it is clear that there is only one certain solution in Eq.(1). In this paper, by Eq. (2) the image will be reconstructed by two 1-D brightness distribution. This method is proposed as accumulation reconstructing method.

\section{Experiment}

In this experiment, we use the above image reconstruction method, and the developed sensor which was constructed from two Obrid-sensors by rotating the angle of each device by 90 degrees as shown in Fig. 7. The results are shown in Fig. 8. Figure 8(a) (d) show the camera image for confirmation. Experimental situations of walk are shown in Fig. 8(a) (c), and the falling is shown in Fig. 8(d). We can see from the Fig. 8(e) (f), the human shape was reconstructed correctly. Although reconstructed images were not clearer than camera images, they were enough for the human shape detection.

\section{Conclusions}

In this paper, a detection of human shape with privacy-preserving by image reconstruction has been developed. The Obrid-sensor can find a person's position from a 1-D brightness distribution without getting 2-D image data. Although this brightness distribution contains enough information to distinguish a person's position, it does not include detailed information about a person. Therefore, the position of a person can be detected correctly without detecting personal detailed features such as facial information. In this work, the developed sensor unit was constructed by two Obrid-sensors which are set horizontally and vertically. The image of human shape was reconstructed by that sensor unit and our proposed method. If we use proposed sensor system, it's easy for everyone to grasp human shape with privacy-preserving without the technical knowledge of the Obrid-sensor.

\section{Acknowledgment}

This work was supported by JSPS KAKENHI Grant Numbers 24700198, 24500670.

\section{References}

(1) I. Kitahara, K. Kogure, and N. Hagita: "Stealth Vision: A Method for Video Capturing System with Protecting Privacyi”, Technical Report of IEICE, Vol. 103, No. 738, pp. 89-94, 2004

(2) T. Koshimizu, T. Toriyama, S. Nishino, and N. Babaguchi, N. Hagita: "Visual Abstraction for Privacy Preserving Video Surveillance”, IEICE Technical Report, Vol. 105, No. 674, pp. 259-302, 2005

(3) K. Yabuta, H. Kitazawa, and T. Tanaka: “A fixed monitoring camera image processing method satisfying both privacy protection and object recognition”, IEICE Technical Report, Vol. 105, No. 29, pp. 13-18, 2005

(4) T. Takenouchi, M. Morimoto, H. Kawahara, M. Takahashi, and H. Yokota: High-Reliability and Compact Passive Infrared Detectors Monitoring Two Independent Areas, Matsushita Elect. Works Tech. Rep., Vol. 52, No. 4, pp. 62-68, 2004

(5) S. Mitsutoshi, Y. Namba, and S. Serikawa: "Extraction of Values Representing Human Features in Order to Develop a Privacy-preserving Sensor”, International Journal of ICIC, Vol. 4, No. 4, pp. 883-895, 2008

(6) M. Hagiwara : "Neuro-Fuzzy Genetic Algorithm”, Sangyo Tosho, 1994

(7) S. Serikawa and T. Simomura: "Proposal of a system of function-discovery using a bug type of artifical life”, IEEJ Trans. on Electronics, Information and Systems, Vol. 118-C, No. 2, pp. 170-179, 1998

(8) S. Serikawa, B. B. Chhetri, and T. Simomura: "Improvement of the search ability of S-System (AFunction-Discovery System)”, IEEJ Trans. on Electronics, Information and Systems, Vol. 120-C, No. 8/9, pp. 1281-1282, 2000

(9) J. Shibata, K. Okuhara, and H. Ishii: “Adaptive worker's arrangement and workload control for projectmanagement by genetic algorithm”, International Journal of Innovative Computing, Information and Control, Vol. 3, No. 1, pp. 175-188, 2007

(10) J. Koza: “Genetic Programming, Auto Discovery of Reusable Subprograms”, MIT Press, 1994 